Proceedings of the Online Conference "Applications of Physics in Mechanical and Material Engineering"

\title{
Buckling Control of Piezolaminated Beams for Compliant Bistable Mechanisms
}

\author{
K. KULińSKI* AND J. PRZYBYLSKI \\ Częstochowa University of Technology, 42-201 Częstochowa, Poland \\ Doi: $10.12693 /$ APhysPolA.139.574 \\ *e-mail: krzysztof .kulinski@pcz.pl
}

\begin{abstract}
This paper is concerned with the effect of axial forces on buckling control of beams with adhesively bonded piezoelectric layers. The type of the system support makes it possible to apply it in compliant mechanisms for precision engineering. In the work, the influence of piezoelectric actuation on buckling behavior of non-uniform Euler-Bernoulli beams with ends restrained against longitudinal displacements is analyzed in detail. It is shown that the in-plane stretching or compressing being a result of the piezoelectric residual stresses may, dependently on the sign of the applied voltage, either counteract or evoke the buckling of the structure. A change in the buckling mode shape for the first critical buckling load with increasing stiffness of elastic foundation is observed.
\end{abstract}

topics: piezoelectric actuation, micro-positioning, buckling, static response enhancement

\section{Introduction}

Researches concerning the buckling behavior of various types of beams, columns and plates have attracted interest from many academic and R\&D centers since 1751 when Leonard Euler proposed a single formula for predicting compressive force causing this type of failure. The overall buckling in structural mechanics results in a sudden transition from one type of deformation shape, observed under pure compression, to the second bent one connected with eccentric compression. Due to an increasing redistribution of internal forces, the buckling leads to instability and collapse of the whole structure or its particular member.

Structural stability as well as dynamic response of slender systems may be enhanced by using, among others, piezoelectric materials and shape memory alloys which have active and controllable mechanical properties. Piezoceramics belong to a special group of materials which convert mechanical energy into electrical energy, or vice versa. Theoretical, numerical and experimental studies of the shape control of slender cantilever beams with piezoelectric patches being under the electric field application were conducted by Schoeftner et al. [1]. Zehetner and Irschik [2] stated that the effect of piezoelectric force induction on the static system response is the most effective for beams with ends fixed in place to prevent longitudinal displacements. The effect of a piezoceramic rod actuation mounted with different offset on the shape control of a slender cantilever column was thoroughly discussed in [3].
In this paper, a passive control by using piezoelectric actuators of the critical buckling capacity of a non-uniform Euler-Bernoulli beam resting on Winkler foundation is discussed. The beam, having one end pinned and the second one guided, is restrained against longitudinal displacements. The inhomogeneity of an analyzed system is a result of symmetrical bonding of a pair of piezoelectric patches to the top and bottom surfaces of the beam. The proposed system due to its unique feature, similarly as a fixed-guided beam studied in [4], may be used as a flexible segment in compliant mechanisms such as bistable mechanisms.

The electric field induction, dependently on the electric field vector sense, generates the in-plane tension or compression in the beam. The main purpose of this study is to investigate how the electric field applied to the piezo actuators may reduce the compressive load to a subcritical level that will eliminate the buckling deformation of the analyzed system.

\section{Problem formulation}

A scheme of an investigated beam with a pair of piezoceramic patches located symmetrically between the beam's ends and resting on Winkler elastic foundation of stiffness modulus $k$ is shown in Fig. 1. The system is subjected to a prescribed end displacement $\delta$ which results in force $P$ whose value may be determined from Hooke's law. The piezosegment is under uniform electric field $\boldsymbol{E}$. 


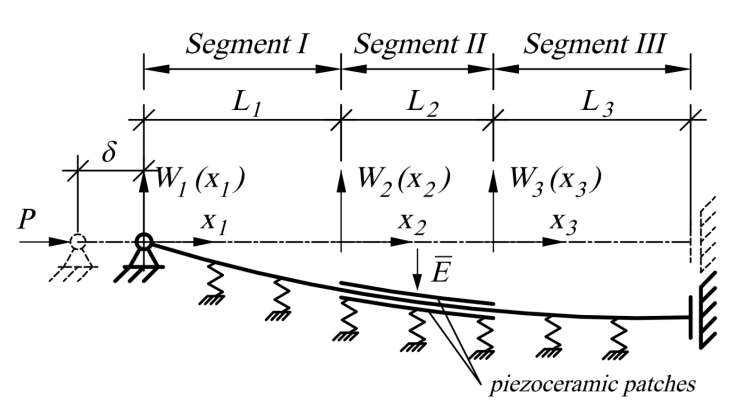

Fig. 1. Scheme of a three-segmented beam with piezo patches located symmetrically between the beam's ends.

The problem is formulated based upon the following assumptions:

- the slenderness of the analyzed system allows to apply the Euler-Bernoulli beam theory,

- the beam is made of a homogeneous linearly elastic isotropic material and the piezoceramic material is linearly elastic and transversely isotropic,

- the system is free of physical and geometrical imperfections,

- segments without piezo elements are identical in terms of mechanical and physical properties,

- the width of piezo patches $\left(b_{\mathrm{p}}=b\right)$ is equal to that of the host beam $\left(b_{\mathrm{b}}=b\right)$ and the piezo layer thickness is considered as $1 / 6$ of the host beam width,

- the adhesive layer between the piezo elements and the host beam is treated as negligibly small,

- delamination of the patches is neglected,

- a uniform and constant electric field is applied to the actuators.

\section{Mathematical solution}

A variational formulation of the problem is based on the linear constitutive equations characterizing piezoelectric material properties:

$$
\begin{gathered}
\sigma_{x}=E_{\mathrm{p}} \varepsilon_{x}-\frac{V}{h_{\mathrm{p}}} e_{31}, \\
D_{z}=e_{31} \varepsilon_{x}+\frac{V}{h_{\mathrm{p}}} \xi_{33},
\end{gathered}
$$

where $E_{\mathrm{p}}$ is the piezoelectric material Young's modulus $\left[\mathrm{N} / \mathrm{m}^{2}\right], \varepsilon_{x}$ is the strain along the considered beam, $e_{31}$ is the piezoelectric constant $\left[\mathrm{C} / \mathrm{m}^{2}\right]$, $V$ denotes the controlling voltage $[\mathrm{V}], h_{\mathrm{p}}$ is the piezoelectric element thickness $[\mathrm{m}], D_{z}$ is the electric displacement $\left[\mathrm{C} / \mathrm{m}^{2}\right]$ and $\xi_{33}$ is the electric permittivity factor $[\mathrm{F} / \mathrm{m}]$.
The problem of residual forces generation in an $n$ segmented beam with $(n-2) / 2$ pairs of piezoceramic actuators, by the assumption that the beam ends eliminate longitudinal displacements, was thoroughly discussed in [5]. On the basis of that study, the residual force for a three-segmented system may be expressed as:

$$
F_{r}= \pm 2 b e_{31} \eta V \text {, }
$$

where its positive or negative value depends on the electric field direction and $\eta$ stands for the relation between the beam and the piezosegment axial stiffness and their lengths:

$$
\eta=\frac{E_{\mathrm{b}} A_{\mathrm{b}} L_{2}}{E_{\mathrm{b}} A_{\mathrm{b}} L_{2}+\left(E_{\mathrm{b}} A_{\mathrm{b}}+E_{\mathrm{p}} A_{\mathrm{p}}\right)\left(L_{1}+L_{3}\right)},
$$

where $E$ is the material Young's modulus, "p" and "b" are the subscripts denoting the piezo element and the host beam, respectively, $A$ is the element cross-section area, and $L_{i}$ is the length of the particular segment $(i=1,2,3)$.

In order to make the problem more general, the analysis is performed with the use of dimensionless quantities. According to that, the boundary value problem for the three-segmented beam resting on elastic foundation with residual force term $\left(f_{r}^{2}\right)$ is expressed by the following differential equations:

$$
\begin{aligned}
& \frac{\mathrm{d}^{4} w_{i}\left(\xi_{i}\right)}{\mathrm{d} \xi_{i}^{4}}+\varphi_{i}\left(p_{u}^{2} \pm f_{r}^{2}\right) \frac{\mathrm{d}^{2} w_{i}\left(\xi_{i}\right)}{\mathrm{d} \xi_{i}^{2}} \\
& +\varphi_{i} \beta w_{i}\left(\xi_{i}\right)=0,
\end{aligned}
$$

for $i=1,2,3$ and for two sets of boundary conditions:

- at the ends of pinned-guided beam

$$
w_{1}(0)=w_{1}^{\mathrm{II}}(0)=w_{3}^{\mathrm{I}}\left(l_{3}\right)=w_{3}^{\mathrm{III}}\left(l_{3}\right)=0,
$$

- the continuity conditions describing the equality of transversal displacements, slopes, bending moments and shear forces at each segment interfaces:

$$
\begin{aligned}
& w_{i}\left(l_{i}\right)=w_{i+1}(0), \quad w_{i}^{\mathrm{I}}\left(l_{i}\right)=w_{i+1}^{\mathrm{I}}(0), \\
& \left(1+r_{m}\right)^{\frac{1}{2}\left(\mathrm{i}^{2 i}+1\right)} w_{i}^{\mathrm{RN}}\left(l_{i}\right)= \\
& \left(1+r_{m}\right)^{\frac{1}{2}\left(\mathrm{i}^{2 i}+1\right)} w_{i+1}^{\mathrm{RN}}(0),
\end{aligned}
$$

where $i=1,2$ and $\mathrm{RN}=\mathrm{II}$, III denotes the derivative order.

The dimensionless parameters are obtained through the following substitutions:

$$
\begin{aligned}
& w_{i}\left(\xi_{i}\right)=W_{i}\left(x_{i}\right) / L, \quad \xi_{i}=x_{i} / L, \\
& \varphi_{i}=\left(1+r_{m}\right)^{-\frac{1}{2}\left(\mathrm{i}^{2 i}+1\right)}, \quad l_{i}=L_{i} / L, \\
& r_{m}=\left(E_{\mathrm{p}} J_{\mathrm{p}}\right) /\left(E_{\mathrm{b}} J_{\mathrm{b}}\right), \quad f_{r}^{2}= \pm v^{2} \eta, \\
& p_{u}^{2}=P_{u} L^{2} /\left(E_{\mathrm{b}} J_{\mathrm{b}}\right), \quad \beta=\left(k L^{4}\right) /\left(E_{\mathrm{b}} J_{\mathrm{b}}\right),
\end{aligned}
$$

where $W_{i}\left(x_{i}\right)$ denotes the transversal displacements of $i$ th segment, $J$ is the element cross-section moment of inertia, $L$ stands for the total beam length, 
$P_{u}$ indicates the axial force resulting from the support prescribed displacement and $k$ is the elastic foundation stiffness modulus. The non-dimensional voltage parameter is defined as:

$$
v=\sqrt{\frac{2 b e_{31} V}{E_{\mathrm{b}} J_{\mathrm{b}}}} L .
$$

Now, the general solution of (5) is taken as follows:

$$
\begin{gathered}
w_{i}\left(\xi_{i}\right)=A_{i} \cosh \left(\gamma_{i 1} \xi_{i}\right)+B_{i} \sinh \left(\gamma_{i 1} \xi_{i}\right) \\
+C_{i} \cosh \left(\gamma_{i 2} \xi_{i}\right)+D_{i} \sinh \left(\gamma_{i 2} \xi_{i}\right),
\end{gathered}
$$

for $i=1,2,3$. Coefficients $\gamma_{i 1}$ and $\gamma_{i 2}$ depend on the axial force sign which depends on prescribed support displacement $\delta$ resulting in force $p_{u}$ and induced residual force parameter $f_{r}$ :

$$
\gamma_{i 1}=\sqrt{\frac{ \pm \varphi_{i}\left(p_{u}^{2}+f_{r}^{2}\right)-\sqrt{\varphi_{i}\left(\left(p_{u}^{2}+f_{r}^{2}\right)^{2}-4 \beta\right)}}{2}}
$$

$$
\gamma_{i 2}=\sqrt{\frac{ \pm \varphi_{i}\left(p_{u}^{2}+f_{r}^{2}\right)+\sqrt{\varphi_{i}\left(p_{u}^{2}+f_{r}^{2}\right)^{2}-4 \varphi_{i} \beta}}{2}} .
$$

By substituting (10) describing the transversal displacements of particular beam segments $(i=$ $1,2,3)$ into the boundary and continuity conditions, (6) and (7), the system of twelve homogeneous linear equations with respect to unknown integration constants $A_{i}, B_{i}, C_{i}$ and $D_{i}$ is obtained. A nontrivial solution of this system is attained, when the determinant of appropriate matrix coefficients is set to zero. As a result, the critical buckling load can be determined for known physical and geometrical parameters of the system as well as the chosen magnitude of the piezoelectric actuation.

\section{Numerical results}

It has been assumed for the computation purposes that the cross-section dimensions of piezoceramic patches and the beam are fractions of the beam's length, hence the width and height of both components $b=B / L=1 / 30$, the beam's height $h_{\mathrm{b}}=H_{\mathrm{b}} / L=0.005$ and the actuator's height $h_{\mathrm{p}}=h_{\mathrm{b}} / 6$. The remaining parameters applied for calculation are equal: $E_{\mathrm{b}} / E_{\mathrm{p}}=0.840$, and $r_{m}=1.63133$. Two elastic foundation parameters are chosen: $\beta=0$ and $\beta=100$. Thus, the buckling load for the beam without piezo patches may be compared with that determined in [6]. Two values of the non-dimensional voltage are adopted: $v \pm \pi$, with the negative voltage inducing the tensile residual force and the positive voltage generating the compressive force, respectively. The influence of piezoelectric actuation on the system's critical force is presented in Fig. 2.

Comparing the courses of curves shown in Fig. 2 one can notice that the longer the piezo patches length, the higher the influence of piezoelectric

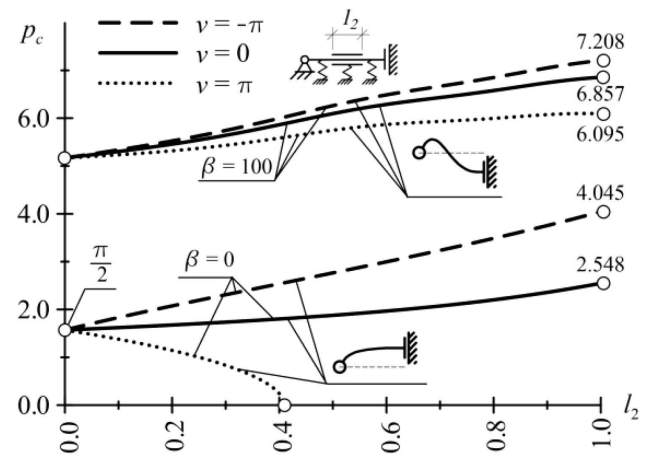

Fig. 2. Two first critical buckling loads $\left(p_{c}\right)$ versus piezo-segment length for different non-dimensional elastic foundation parameters.

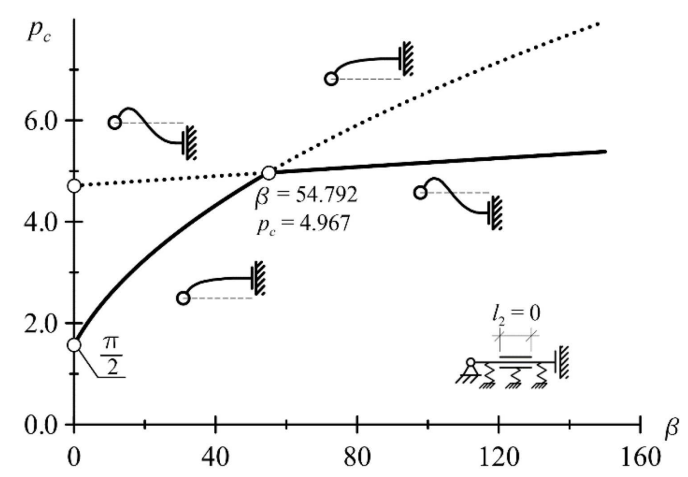

Fig. 3. Modification of buckling modes with increasing values of the elastic foundation modulus for a pinned-guided beam. Remaining parameters: $f=0, l_{2}=0$.

actuation on the critical loads. For the beam without distributed support resulting from the elastic foundation $(\beta=0)$, when the piezosegment length is greater than 0.42 , the positive voltage application of the value $v=\pi$ leads to the buckling instability. The tensile force induction by the piezo actuation in any studied configurations allows one to increase the critical force, however, the higher the elastic foundation stiffness, the lower the influence of residual force on the static response. For $\beta=0$ and $l_{2}=1.00$, the induction of the tensile residual force by $v=\pi$ gives the percentage increase in the critical load equal to $\Delta p_{c}=58.75 \%$, whereas for the beam resting on elastic foundation $(\beta=100)$ and the same piezosegment length $\left(l_{2}=1.00\right)$, one obtains the increase $\Delta p_{c}=5.11 \%$, respectively.

With an increase of the foundation modulus parameter, a change in the buckling modes is detected, what was revealed by Hetenyi [7] for a pinnedpinned beam. In the analyzed system, regardless of the piezosegment length and the actuation level, for $\beta=0$, the first buckling mode without a node is observed, whereas for $\beta=100$, a one-node shape appears. The change of buckling mode shapes for the growing values of elastic foundation stiffness $\beta$ for a simple pinned/guided supported beam $\left(l_{2}=0\right)$ is shown in Fig. 3. 
The numerical results prove that for $\beta=54.792$, one obtains a change in a buckling mode shape for the first critical load. In the case of the configurations with actuators, the piezo elements increase the beam overall bending stiffness, thus a change between modes appears for higher values of the $\beta$ parameter.

\section{Conclusions}

The static response enhancement via piezoelectric actuation of a slender pinned-guided system resting on a Winkler elastic foundation is studied. Regardless of the elastic foundation parameter, the induction of the tensile axial residual force leads to an increase in the critical buckling force. Moreover, depending on the foundation stiffness, a change in the buckling mode shapes for the first critical load is demonstrated.

\section{References}

[1] J. Schoeftner, G. Buchberger, A. Brandl, H. Irschik, Comp. Struct. 133, 746 (2015).

[2] C. Zehetner, H. Irschik, Smart Struct. Syst. 4, 67 (2008).

[3] J. Przybylski, K. Kuliński, MSSP 148, 1 (2021).

[4] G.L. Holst, G.H. Teichert, B.D. Jensen, J. Mech. Design 133, 1 (2011).

[5] J. Przybylski, Eng. Struct. 30, 3739 (2008).

[6] A. Eryilmaz, M.T. Atay, S.B. Coşkun, M. Başbük, App. Math. 2013, 341063 (2013).

[7] M. Hetenyi, Beams on Elastic Foundation, Michigan 1971. 